\title{
STRATIFICATIONS OF ALGEBRAS WITH TWO SIMPLE MODULES
}

\author{
QUNHUA LIU AND DONG YANG
}

\begin{abstract}
Let $A$ be a finite-dimensional algebra with two simple modules. It is shown that if the derived category of $A$ admits a stratification with simple factors being the base field $k$, then $A$ is derived equivalent to a quasi-hereditary algebra. As a consequence, if further $k$ is algebraically closed and $A$ has finite global dimension, then $A$ is either derived simple or derived equivalent to a quasi-hereditary algebra.
\end{abstract}

MSC 2010 classification: 16E35, 16E40, 16E45.

Keywords: recollement, stratification, quasi-hereditary algebra, derived simple algebra, Hochschild cohomology.

\section{INTRODUCTION}

Quasi-hereditary algebras, introduced by Cline, Parshall and Scott [5, 20], are finite-dimensional algebras with certain ring-theoretical stratifications, which induce stratifications of their derived categories with simple factors being finite-dimensional division algebras, due to [6]. One asks if the converse is true, roughly speaking, if a stratification of the derived category induces a ring-theoretical stratification of the algebra. This can possibly be true only up to derived equivalence, because the class of quasi-hereditary algebras is not closed under derived equivalence, see [7, Example] and Section 2.4 for examples.

Question 1.1. Let $k$ be a field and $A$ be a finite-dimensional $k$-algebra. Assume that $\mathcal{D}(\operatorname{Mod} A)$ admits a stratification with simple factors being finite-dimensional division k-algebras. Is $A$ then derived equivalent to a quasi-hereditary algebra?

In this paper, we give a partial answer to the question for $A$ with two (isomorphism classes of) simple modules.

Theorem $1.2(=3.6)$. Let $k$ be a field and let $A$ be a finite-dimensional $k$-algebra with two simples modules. If $\mathcal{D}(\operatorname{Mod} A)$ admits a stratification with all simple factors being $k$, then $A$ is derived equivalent to a quasi-hereditary algebra.

The key point is to prove that a certain differential graded algebra, which is derived equivalent to $A$, has cohomologies concentrated in two successive degrees. This is achieved by comparing Hochschild cohomologies. As a consequence of Theorem 1.2, there is the following dichotomy, which was used in the introduction of [18] but was not proved in the literature.

Date: Last modified on March 25, 2022.

The first named author acknowledges partial support by Jiangsu Province BK20130899. Both authors acknowledge partial support by Natural Science Foundation of China 11301272. They thank a referee for helpful suggestions. 
Theorem 1.3 (=3.7). Let $k$ be an algebraically closed field and let $A$ be a finite-dimensional $k$-algebra with two simple modules. Assume that $A$ has finite global dimension. Then $A$ is either derived simple or derived equivalent to a quasi-hereditary algebra.

Theorems 1.2 and 1.3 are proved in Section 3. In Section 4 we illustrate by an example how Theorem 1.3 is applied to show the derived simplicity of algebras. More precisely, we construct a family of algebras $A_{n}(\underline{x}, \underline{y})$ with two simple modules for $n \geq 0$ and two sequences of positive integers $\underline{x}, \underline{y}$, which extends the family of algebras $A_{n}$ studied by E. Green [10], Happel [13] and Membrillo-Hernández [18]. We show, by comparing the top Hochschild cohomologies, that $A_{n}(\underline{x}, \underline{y})$ for any $n \geq 3$ and any $\underline{x}, \underline{y}$ is not derived equivalent to a quasi-hereditary algebra and hence is derived simple.

Throughout, let $k$ be a field. All algebras and modules are over $k$. We use right modules.

\section{Preliminaries}

In this section, we recall the definitions and basic properties of derived categories of differential graded algebras, Hochschild cohomologies, recollements and stratifications. We also recall the classification of quasi-hereditary algebras with two simple modules.

2.1. Derived category. We follow [15, 16]. For a differential graded (=dg) algebra $A$, let $\mathcal{D}(A)$ denote the derived category of (right) dg $A$-modules. If $A$ is an (ordinary) algebra, considered as a dg algebra concentrated in degree 0 , then a $\mathrm{dg} A$-module is nothing else but a complex of (right) $A$-modules and $\mathcal{D}(A)$ is the same as $\mathcal{D}(\operatorname{Mod} A)$, the unbounded derived category of the abelian category $\operatorname{Mod} A$ of $A$-modules.

For two $\operatorname{dg} A$-modules $M$ and $N$, define $\mathcal{H o m}_{A}(M, N)$ to be the complex whose component $\mathcal{H o m}_{A}^{p}(M, N)$ consists of homogenous maps of graded $A$-modules of degree $p$ from $M$ to $N$, i.e.

$$
\mathcal{H} \operatorname{om}_{A}^{p}(M, N)=\left\{f \in \prod_{q \in \mathbb{Z}} \operatorname{Hom}_{k}\left(M^{q}, N^{p+q}\right) \mid f(m a)=f(m) a, \forall m \in M \text { and } a \in A\right\},
$$

and whose differential is given by $d(f)=d_{N} f-(-1)^{p} f d_{M}$ for $f \in \mathcal{H} \circ m_{A}^{p}(M, N)$. The complex $\mathcal{E n d}_{A}(M)=\mathcal{H} \operatorname{om}_{A}(M, M)$ is a dg algebra with multiplication being the composition of maps. For any $\operatorname{dg} A$-module $M$, by [15, Theorem 3.1], there is a cofibrant dg $A$-module $P$ together with a quasi-isomorphism $P \rightarrow M$ of $\operatorname{dg} A$-modules (called the cofibrant resolution of $M$ ). In this case, there is a natural isomorphism for all $p \in \mathbb{Z}$

$$
\operatorname{Hom}_{\mathcal{D}(A)}(M, N[p]) \cong H^{p} \mathcal{H o m} A(P, N) .
$$

We refer to [17, Section 2.12] for the definition of cofibrant dg module and remark that if $A$ is an algebra then a bounded complex of finitely generated projective $A$-modules is a cofibrant $\mathrm{dg}$ $A$-module.

A dg $A$-module $M$ is compact in $\mathcal{D}(A)$ if $M$ belongs to $\operatorname{per}(A)=\operatorname{thick}\left(A_{A}\right)$, the smallest triangulated subcategory of $\mathcal{D}(A)$ which contains $A_{A}$ and which is closed under taking direct summands. It is a compact generator of $\mathcal{D}(A)$ if $\operatorname{thick}(M)=\operatorname{per}(A)$. In the case when $A$ is an algebra, $\operatorname{per}(A)$ is triangle equivalent to $K^{b}(\operatorname{proj} A)$, the homotopy category of bounded complexes of finitely generated projective $A$-modules. If $M$ is a cofibrant $\operatorname{dg} A$-module and is a 
compact generator of $\mathcal{D}(A)$, then by [15, Lemma 6.1 (a)] the two dg algebras $\mathcal{E} n d_{A}(M)$ and $A$ are derived equivalent, namely, there is a triangle equivalence

$$
? \stackrel{L}{\otimes} \mathcal{E}_{d_{A}(M)} M: \quad \mathcal{D}\left(\mathcal{E} n d_{A}(M)\right) \stackrel{\simeq}{\longrightarrow} \mathcal{D}(A) .
$$

Further, two quasi-isomorphic dg algebras are derived equivalent.

2.2. Hochschild cohomology. We follow [14. Let $A$ be a $\operatorname{dg}$ algebra. Then $A$ is a $\operatorname{dg} A$ bimodule, in other words, $A$ is a dg module over the enveloping algebra $A^{e}:=A^{o p} \otimes_{k} A$. For an integer $p$, the $p$-th Hochschild cohomology of $A$ is defined as

$$
H H^{p}(A):=\operatorname{Hom}_{\mathcal{D}\left(A^{e}\right)}(A, A[p]) .
$$

If $A$ is a finite-dimensional algebra over the field $k$, then $H H^{p}(A)=\operatorname{Ext}_{A^{e}}^{p}(A, A)$, which vanishes for $p<0$ and for $p>\operatorname{gl} . \operatorname{dim} A$ (see [12, Section 1.5]).

Let $A$ be an algebra and $B$ be a dg algebra which is derived equivalent to $A$. Then by $[15$, Section 9], there is a dg $B$-A-bimodule $M$ such that $? \stackrel{L}{\otimes_{B}} M: \mathcal{D}(B) \rightarrow \mathcal{D}(A)$ is a triangle equivalence. It follows from [14, Corollary 3.2] that there is an isomorphism for all $p \in \mathbb{Z}$

$$
H H^{p}(A) \cong H H^{p}(B) .
$$

2.3. Recollement and stratification. A recollement of derived categories of algebras is a diagram of triangulated functors

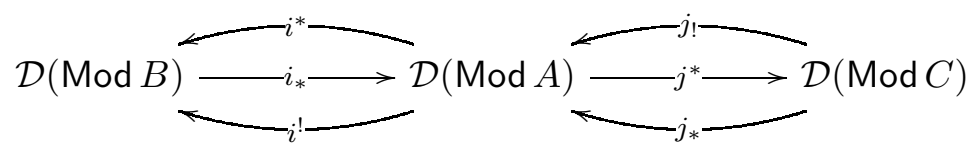

satisfying certain conditions, where $A, B, C$ are algebras. This notion was introduced by Beilinson, Bernstein and Deligne in [4] and can be considered as a short exact sequence of derived categories. We refer to [4] for the precise conditions and provide only a few properties for later use. For a finite-dimensional algebra $A$, let $r(A)$ denote the number of isomorphism classes of simple $A$-modules.

Lemma 2.1. (4, 2]) Suppose there is a recollement of the form [2.1). The following hold true:

(a) $i_{*}$ and $j_{!}$are full embeddings and $\operatorname{Hom}\left(j_{!}(X), i_{*}(Y)\right)=0$ for any $X \in \mathcal{D}(\operatorname{Mod} C)$ and $Y \in \mathcal{D}(\operatorname{Mod} B)$.

(b) A has finite global dimension if and only if so do $B$ and $C$.

(c) If $A$ is finite-dimensional, then so are $B$ and $C$. In this case, $r(A)=r(B)+r(C)$.

(d) If $A$ has finite global dimension, then $j_{!}(C) \oplus i_{*}(B)$ is a compact generator of $\mathcal{D}(\operatorname{Mod} A)$.

The recollement (2.1) is non-trivial if both $B$ and $C$ are non-trivial. An algebra $A$ is derived simple if there are no non-trivial recollements of $\mathcal{D}(\operatorname{Mod} A)$ of the form (2.1). For example, a division algebra is derived simple.

If $A$ is not derived simple, then there is a non-trivial recollement of $\mathcal{D}(\operatorname{Mod} A)$ of the form (2.1). If further $B$ and/or $C$ are not derived simple, we can then take a non-trivial recollement of $\mathcal{D}(\operatorname{Mod} B)$ and/or $\mathcal{D}(\operatorname{Mod} C)$. Continuing this process with every occurring algebra unless it is derived simple, eventually we obtain a stratification of $\mathcal{D}(\operatorname{Mod} A)$, which is analogous to 
composition series. The derived simple algebras obtained in this process are the simple factors of the stratification.

Typical examples of stratifications are given for quasi-hereditary algebras ([5, 20]). Let $A$ be a finite-dimensional algebra and $e \in A$ an idempotent. The ideal $A e A$ is a heredity ideal if $e A e$ is semisimple and $A e A$ is projective as a right $A$-module. Applying [6, Corollary 3.7] and [2, Proposition 4.1], we obtain a recollement

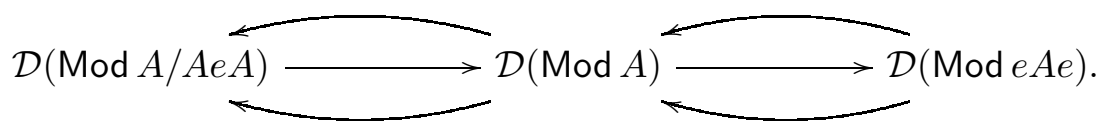

The algebra $A$ is quasi-hereditary if there is a heredity chain, i.e. a finite chain of ideals

$$
0=J_{0} \subseteq J_{1} \subseteq \ldots \subseteq J_{m}=A
$$

such that $J_{i} / J_{i-1}$ is a heredity ideal of $A / J_{i-1}$ for all $1 \leq i \leq m$. We can refine this chain such that in each step the semisimple algebra associated to the heredity ideal is simple. Then by repeating the above construction, we obtain a stratification of $\mathcal{D}(\operatorname{Mod} A)$.

2.4. Quasi-hereditary algebras with two simple modules. Let $x, y \geq 0$ be integers. Let $Q(x, y)$ be the quiver which has two vertices 1 and 2 and which has $x$ arrows $\alpha_{1}, \ldots, \alpha_{x}$ from 1 to 2 and $y$ arrows $\beta_{1}, \ldots, \beta_{y}$ from 2 to 1 . Let $B(x, y)$ be the quotient of the path algebra of $Q(x, y)$ by the ideal generated by the paths $\beta_{q} \alpha_{p}$ for $1 \leq p \leq x$ and $1 \leq q \leq y$. It is easy to check that the global dimension of $B(x, y)$ is at most 2 and that $B(x, y)$ is quasi-hereditary with heredity chain

$$
0 \subseteq B(x, y) e_{1} B(x, y) \subseteq B(x, y)
$$

Conversely, any quasi-hereditary algebra $A$ with two simple modules whose endomorphism algebras are $k$ is Morita equivalent to some $B(x, y)$, see [18, Theorem 3.1].

We remark that $B(x, y)$ and $B(y, x)$ are Ringel dual to each other, i.e. $B(y, x)$ is the endomorphism algebra of the characteristic tilting module over $B(x, y)$ (see [19] for the definition of the characteristic tilting module). The proof is direct and is left to the reader as an exercise. A consequence of this fact is that $B(x, y)$ and $B(y, x)$ are derived equivalent.

Next we show that, when $x \geq 2$ and $y \geq 1$, the quasi-hereditary algebra $B(x, y)$ is derived equivalent to an algebra which is not quasi-hereditary. So the class of quasi-hereditary algebras with two simples is not closed under derived equivalence.

Let $P_{1}$ and $P_{2}$ be the indecomposable projective $B(x, y)$-module at vertices 1 and 2 , respectively. Consider the map

$$
f=\left(\alpha_{1}, \ldots, \alpha_{x}\right)^{\operatorname{tr}}: P_{1} \longrightarrow P_{2}^{\oplus x} .
$$

Let $T=\operatorname{cok}(f) \oplus P_{2}$. It is straightforward to check that $T$ is a tilting module over $B(x, y)$. (Alternatively, $T$ is the left silting mutation of $P_{1} \oplus P_{2}$ at $P_{1}$ in the sense of Aihara-Iyama [1]. It follows that $T$ is self-orthogonal and hence is a tilting module.) The endomorphism algebra $E$ of $T$ is the quotient of the path algebra of the quiver which has two vertices 1 and 2 and which has $x^{2} y$ arrows $a_{r}^{p q}(1 \leq p, q \leq x, 1 \leq r \leq y)$ from 1 to 2 and $x$ arrows $b_{p}(1 \leq p \leq x)$ from 2 to 1 modulo the ideal generated by the relations $\sum_{q=1}^{x} b_{q} a_{r}^{p q}(1 \leq p \leq x, 1 \leq r \leq y), a_{r}^{p q} b_{p}=a_{r}^{p^{\prime} q} b_{p^{\prime}}$ 
$\left(1 \leq p, q, p^{\prime} \leq x, 1 \leq r \leq y\right)$ and $a_{r}^{p q} b_{p^{\prime}}\left(1 \leq p, q, p^{\prime} \leq x\right.$ with $\left.p \neq p^{\prime}, 1 \leq r \leq y\right)$. It is readily seen that $E$ is not isomorphic to any $B\left(x^{\prime}, y^{\prime}\right)$, and hence is not quasi-hereditary.

\section{The MAIN RESUlts}

In this section, we prove Theorems 1.2 and 1.3 . We show that a finite-dimensional algebra, which has two simples and whose derived category admits a stratification with simple factors being the base field $k$, is derived equivalent to a certain graded $n$-Kronecker algebra, which is in turn derived equivalent to a quasi-hereditary algebra. The grading of this $n$-Kronecker algebra is determined by comparing Hochschild cohomologies. We start with computing the Hochschild cohomologies of graded $n$-Kronecker algebras.

3.1. The graded n-Kronecker algebra: Hochschild cohomologies. Let $n \geq 1$ be a positive integer. Let $Q$ be a graded $n$-Kronecker quiver, namely, the vertex set $Q_{0}$ consists of two vertices 1 and 2 , the arrow set $Q_{1}$ consists of $n$ arrows, which are all from 1 to 2 , and to each arrow we assign an integer. The corresponding graded path algebra $\Lambda=k Q$ is called a graded $n$-Kronecker algebra. We write $V=\bigoplus_{p \in \mathbb{Z}} V^{p}$ for the graded vector space of arrows of $Q$. Thus $V^{p}$ has a basis formed by arrows of $Q$ of degree $p$. Then $\Lambda=k Q_{0} \oplus V$. Written as a matrix algebra, $\Lambda$ has the form

$$
\Lambda=\left(\begin{array}{ll}
k & 0 \\
V & k
\end{array}\right) .
$$

Our aim in this subsection is to calculate the dimension of the Hochschild cohomologies $H H^{p}(\Lambda)$ of $\Lambda$, viewed as a dg algebra with trivial differential. More precisely, we show the following result.

Proposition 3.1. Let $Q$ be a graded $n$-Kronecker quiver and $\Lambda=k Q_{0} \oplus V$ its graded path algebra. Then

$$
\operatorname{dim}_{k} H H^{p}(\Lambda)= \begin{cases}\#\left\{(\alpha, \beta) \in Q_{1} \times Q_{1} \mid \operatorname{deg} \alpha-\operatorname{deg} \beta=0\right\}-1 & \text { if } p=1 \\ \#\left\{(\alpha, \beta) \in Q_{1} \times Q_{1} \mid \operatorname{deg} \alpha-\operatorname{deg} \beta=-1\right\}+1 & \text { if } p=0 \\ \#\left\{(\alpha, \beta) \in Q_{1} \times Q_{1} \mid \operatorname{deg} \alpha-\operatorname{deg} \beta=p-1\right\} & \text { if } p \neq 0,1 .\end{cases}
$$

We give two corollaries before presenting the proof.

Corollary 3.2. Let $\Lambda$ and $V$ be the same as in Proposition 3.1,

(1) The equality $\operatorname{dim}_{k} H H^{p}(\Lambda)=\operatorname{dim}_{k} H H^{2-p}(\Lambda)$ holds for all $p \neq 0,1$. And $\operatorname{dim}_{k} H H^{0}(\Lambda)=$ $\operatorname{dim}_{k} H H^{2}(\Lambda)+1$.

(2) $\sum_{p \in \mathbb{Z}} \operatorname{dim}_{k} H H^{p}(\Lambda)=\left(\operatorname{dim}_{k} V\right)^{2}=n^{2}$.

(3) Let $a$ and $b$ be the minimal and the maximal degrees of arrows of $Q$, respectively. Then $H H^{p}(\Lambda)=0$ unless $p \in[1-b+a, 1+b-a] \cup\{0\}$. Moreover, $H H^{1-b+a}(\Lambda) \neq 0$ if $1-b+a \leq 0$.

Proof. These follow straightforward from Proposition 3.1 .

Corollary 3.3. Let $\Lambda$ and $V$ be the same as in Proposition 3.1. If $\Lambda$ is derived equivalent to an algebra, then $V$ is concentrated in at most two successive degrees. 
Proof. If $\Lambda$ is derived equivalent to an algebra, then $H H^{p}(\Lambda)$ vanishes for all $p<0$. By Corollary 3.2 (3), the latter condition implies $1-b+a \geq 0$, namely $b-a \leq 1$, which, by the definition of $a$ and $b$, means exactly that $V$ is concentrated in at most two successive degrees.

Now we provide a proof of Proposition 3.1, followed by two examples as illustration.

Proof of Proposition 3.1. Recall that $\Lambda^{e}$ stands for the enveloping algebra $\Lambda^{\mathrm{op}} \otimes_{k} \Lambda$ and the $p$-th Hochschild cohomology $H H^{p}(\Lambda)$ is defined to be $\operatorname{Hom}_{\mathcal{D}\left(\Lambda^{e}\right)}(\Lambda, \Lambda[p])$. We stress that here $\Lambda$ and $\Lambda^{e}$ are viewed as dg algebras with trivial differential and $\mathcal{D}\left(\Lambda^{e}\right)$ is the derived category of $\mathrm{dg}$ $\Lambda^{e}$-modules.

First we construct a cofibrant resolution $P$ of $\Lambda$ as $\operatorname{dg} \Lambda^{e}$-module by using the Koszul bimodule resolution of $\Lambda$. We claim that the following complex is exact and is a projective resolution of $\Lambda$ as graded $\Lambda^{e}$-module:

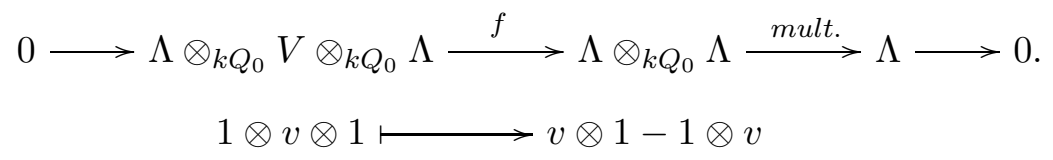

Indeed, by [21, Section 3], this is a projective resolution of $\Lambda$ as $\Lambda^{e}$-module if we forget the grading. Therefore by definition $P:=$ Cone $(f)$, where $f$ is viewed as a homomorphism of $\mathrm{dg}$ $\Lambda^{e}$-modules, is cofibrant over $\Lambda^{e}$ and hence is a cofibrant resolution of $\Lambda$.

It follows that

$$
H H^{p}(\Lambda) \cong \operatorname{Hom}_{\mathcal{D}\left(\Lambda^{e}\right)}(\Lambda, \Lambda[p]) \cong H^{p} \mathcal{H} o m_{\Lambda^{e}}(P, \Lambda) .
$$

Now $P$ is the mapping cone of $f$, hence

$$
\mathcal{H}_{o m} \Lambda^{e}(P, \Lambda) \cong \mathcal{H} o m_{\Lambda^{e}}(\operatorname{Cone}(f), \Lambda) \cong \operatorname{Cone}\left(\mathcal{H} o m_{\Lambda^{e}}(f, \Lambda)\right)[-1] .
$$

Further observe the isomorphisms $\Lambda \otimes_{k Q_{0}} V \otimes_{k Q_{0}} \Lambda \cong V \otimes_{k Q_{0}^{e}} \Lambda^{e}$ and $\Lambda \otimes_{k Q_{0}} \Lambda \cong \Lambda \otimes_{k Q_{0}}$ $k Q_{0} \otimes_{k Q_{0}} \Lambda \cong k Q_{0} \otimes_{k Q_{0}^{e}} \Lambda^{e}$. So the map $f^{*}:=\mathcal{H}_{o m_{\Lambda}}(f, \Lambda)$ can be calculated explicitly:

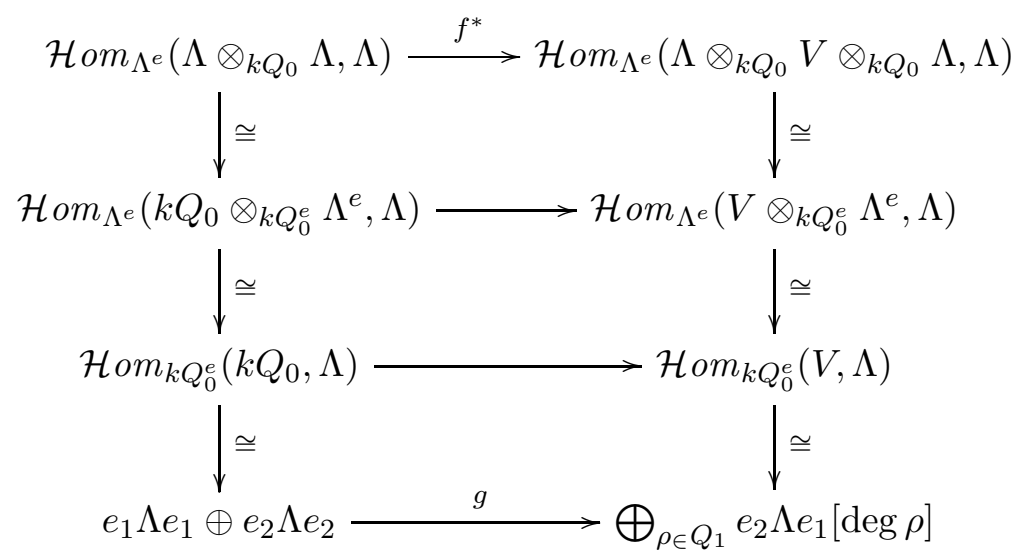

where $e_{1}$ and $e_{2}$ are the two primitive idempotents of $\Lambda$. The middle vertical isomorphisms are obtained by adjunction. The lower vertical isomorphisms are obtained by observing the two isomorphisms of graded $k Q_{0}^{e}$-modules: $V=e_{2} \Lambda e_{1} \cong \bigoplus_{\rho \in Q_{1}}\left(\left(e_{2} \otimes e_{1}\right) k Q_{0}^{e}\right)[-\operatorname{deg} \rho]$ and $k Q_{0}=\left(e_{1} \otimes e_{1}\right) k Q_{0}^{e} \oplus\left(e_{2} \otimes e_{2}\right) k Q_{0}^{e}$. We have the formula

$$
\operatorname{dim}_{k}\left(e_{2} \Lambda e_{1}[\operatorname{deg} \rho]\right)^{p}=\#\left\{\alpha \in Q_{1} \mid \operatorname{deg} \alpha-\operatorname{deg} \rho=p\right\} .
$$


So

$$
\operatorname{dim}_{k}\left(\bigoplus_{\rho \in Q_{1}} e_{2} \Lambda e_{1}[\operatorname{deg} \rho]\right)^{p}=\#\left\{(\alpha, \beta) \in Q_{1} \times Q_{1} \mid \operatorname{deg} \alpha-\operatorname{deg} \beta=p\right\} .
$$

Finally, the map $g$ in the last row is given by $g\left(e_{1}\right)=(\rho[\operatorname{deg} \rho])_{\rho \in Q_{1}}=-g\left(e_{2}\right)$. In particular, both the image and the kernel of $g$ are one-dimensional. Since both the domain and the codomain of $g$ have trivial differentials, the desired formula follows.

Example 3.4. Suppose that we have a graded 3-Kronecker quiver with $Q_{1}=\left\{\alpha_{-1}, \alpha_{0}, \alpha_{2}\right\}$ and $\operatorname{deg} \alpha_{p}=p$ for $p=-1,0,2$. Then $V=V^{-1} \oplus V^{0} \oplus V^{2}$, where each $V^{p}$ is one-dimensional with basis $\alpha_{p}$. In this case we have $\mathcal{H}_{\mathrm{om}_{k} \mathrm{Q}_{0}^{e}}(V, \Lambda) \cong V[-1] \oplus V \oplus V[2]$.It follows that

$$
\operatorname{dim}_{k} H H^{p}(\Lambda)= \begin{cases}1 & p=-2,-1,2,3,4 \\ 2 & p=0 \\ 2 & p=1 \\ 0 & \text { otherwise. }\end{cases}
$$

Example 3.5. Let $x, y \geq 0$ be integers such that $x+y \geq 1$. Suppose that we have a graded $n$-Kronecker quiver with $n=x+y, Q_{1}=\left\{\alpha_{0,1}, \ldots, \alpha_{0, y}, \alpha_{1,1}, \ldots, \alpha_{1, x}\right\}$ and $\operatorname{deg} \alpha_{p, q}=p$. Then $V=V^{0} \oplus V^{1}$, where $V^{0}$ is $y$-dimensional with basis $\left\{\alpha_{0,1}, \ldots, \alpha_{0, y}\right\}$, and $V^{1}$ is $x$-dimensional with basis $\left\{\alpha_{1,1}, \ldots, \alpha_{1, x}\right\}$. Here $\mathcal{H o m}_{k Q_{0}^{e}}(V, \Lambda) \cong V^{\oplus y} \oplus V[1]^{\oplus x}$. It follows that

$$
\operatorname{dim}_{k} H H^{p}(\Lambda)= \begin{cases}x y+1 & p=0 \\ x^{2}+y^{2}-1 & p=1 \\ x y & p=2 \\ 0 & \text { otherwise. }\end{cases}
$$

\subsection{The main results.}

Theorem 3.6. Let $A$ be a finite-dimensional k-algebra with two simple modules. Assume that $\mathcal{D}(\operatorname{Mod} A)$ admits a stratification with all simple factors being $k$. Then $A$ is derived equivalent to a quasi-hereditary algebra.

Proof. First we show that such an algebra $A$ must be derived equivalent to a graded $n$-Kronecker algebra $\Lambda$. By Lemma 2.1 (c), a stratification of $\mathcal{D}(\operatorname{Mod} A)$ has precisely two simple factors, namely, it is a recollement of the form

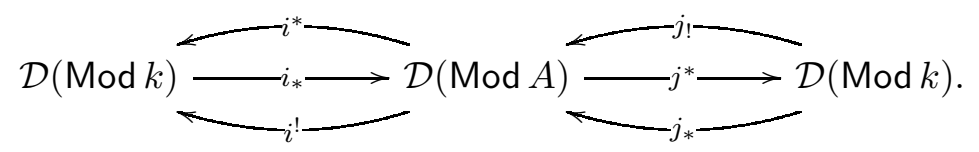

Take $T=i_{*}(B) \oplus j_{!}(C) \in \mathcal{D}(\operatorname{Mod} A)$. By Lemma2.1 $(\mathrm{d}), T$ is a compact generator of $\mathcal{D}(\operatorname{Mod} A)$. We may assume that $T$ is a bounded complex of finitely generated projective $A$-modules, in particular, it is a cofibrant $\mathrm{dg} A$-module. Then $A$ is derived equivalent to the $\mathrm{dg}$ endomorphism algebra $\tilde{\Lambda}=\mathcal{E} n d_{A}(T)$. Written as a matrix algebra, $\tilde{\Lambda}$ has the form

$$
\tilde{\Lambda}=\left(\begin{array}{cc}
\mathcal{E} n d_{A}\left(i_{*}(k)\right) & \mathcal{H} o m_{A}\left(j_{!}(k), i_{*}(k)\right) \\
\mathcal{H} \circ m_{A}\left(i_{*}(k), j_{!}(k)\right) & \mathcal{E} n d_{A}\left(j_{!}(k)\right)
\end{array}\right) .
$$


Now by Lemma 2.1 (a), the space $\operatorname{Hom}_{\mathcal{D}(\operatorname{Mod} A)}\left(i_{*}(k), i_{*}(k)[p]\right)$ vanishes if $p \neq 0$ and is $k$ if $p=0$. Therefore, since $i_{*}(k)$ is cofibrant over $A$, the dg algebra $\mathcal{E} n d_{A}\left(i_{*}(k)\right)$ is quasi-isomorphic to $k$. It follows that the embedding $k \cong k\left\{\operatorname{id}_{i_{*}(k)}\right\} \hookrightarrow \mathcal{E} n d_{A}\left(i_{*}(k)\right)$ is a quasi-isomorphism of dg algebras. For the same reason, the embedding $k \cong k\left\{\operatorname{id}_{j_{1}(k)}\right\} \hookrightarrow \mathcal{E} n d_{A}\left(j_{!}(k)\right)$ is a quasi-isomorphism of $\mathrm{dg}$ algebras, and similarly, the complex $\mathcal{H o m}_{A}\left(j_{!}(k), i_{*}(k)\right)$ is acyclic. Further, we can take a subcomplex $V$ of $\mathcal{H o m}_{A}\left(i_{*}(k), j_{!}(k)\right)$ such that the embedding $V \hookrightarrow \mathcal{H} o m_{A}\left(i_{*}(k), j_{!}(k)\right)$ is a quasi-isomorphism of complexes of vector spaces (thus as a graded vector space $V$ is isomorphic to $\left.\bigoplus_{p \in \mathbb{Z}} \operatorname{Hom}_{\mathcal{D}(\operatorname{Mod} A)}\left(i_{*}(k), j_{!}(k)[p]\right)\right)$. It follows that the embedding

$$
\Lambda=\left(\begin{array}{cc}
k & 0 \\
V & k
\end{array}\right) \hookrightarrow \tilde{\Lambda}
$$

is a quasi-isomorphism of dg algebras. Therefore, $\Lambda$, viewed as a dg algebra with trivial differential, is derived equivalent to $\tilde{\Lambda}$, and hence it is derived equivalent to $A$.

Now by Corollary 3.3 the graded vector space $V$ is concentrated in at most two successive degrees. We can assume that $V$ is concentrated in degrees 0 and 1, i.e. $V=V^{0} \oplus V^{1}$, by shifting $i_{*}(B)$ in the definition of $T$ if necessary.

Next we consider the quasi-hereditary algebra $B=B(x, y)$ for $x=\operatorname{dim}_{k} V^{1}$ and $y=\operatorname{dim}_{k} V^{0}$ (see Section 2.4). The derived category $\mathcal{D}(\operatorname{Mod} B)$ admits a recollement of the form (3.1), and here $i_{*}(k)=B / B e_{1} B$ and $j_{!}(k)=e_{1} B$. Direct computation shows that $\operatorname{Ext}_{B}^{p}\left(B / B e_{1} B, e_{1} B\right) \neq 0$ only for $p=0$ and 1; Moreover, $\operatorname{dim}_{k} \operatorname{Hom}_{B}\left(B / B e_{1} B, e_{1} B\right)=y$ and $\operatorname{dim}_{k} \operatorname{Ext}_{B}^{1}\left(B / B e_{1} B, e_{1} B\right)=$ $x$. Therefore, we have isomorphisms of graded vector spaces

$$
\bigoplus_{p \in \mathbb{Z}} \operatorname{Hom}_{\mathcal{D}(\operatorname{Mod} B)}\left(B / B e_{1} B, e_{1} B[p]\right) \cong \bigoplus_{p \in \mathbb{Z}} \operatorname{Ext}_{B}^{p}\left(B / B e_{1} B, e_{1} B\right) \cong k^{\oplus y} \oplus k^{\oplus x}[-1] .
$$

Applying the above argument, one sees that $B$ is derived equivalent to the graded $n$-Kronecker algebra

$$
\left(\begin{array}{cc}
k & 0 \\
k^{\oplus y} \oplus k^{\oplus x}[-1] & k
\end{array}\right) .
$$

This is precisely the algebra $\Lambda$ we have constructed above.

To summarise, the algebra $A$ is derived equivalent to a graded $n$-Kronecker algebra, which is in turn derived equivalent to a quasi-hereditary algebra $B$. We are done.

Next we prove the following dichotomy.

Theorem 3.7. Let $k$ be algebraically closed and $A$ be a finite-dimensional k-algebra with two simple modules. Assume that $A$ has finite global dimension. Then $A$ is either derived simple or derived equivalent to a quasi-hereditary algebra.

Proof. Suppose that $A$ is not derived simple. Then the derived category $\mathcal{D}(\operatorname{Mod} A)$ admits a recollement

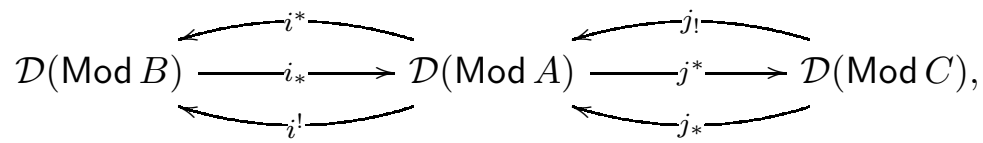


where $B$ and $C$ are non-trivial algebras. It follows from Lemma 2.1 that $B$ and $C$ are finitedimensional local algebras of finite global dimension. Thus $B$ and $C$ are Morita equivalent to $k$, since the base field $k$ is algebraically closed. Without loss of generality we may assume that $B$ and $C$ are the field $k$. As a consequence, the above recollement is a stratification of $\mathcal{D}(\operatorname{Mod} A)$ with simple factors being $k$. By Theorem 3.6, $A$ is derived equivalent to a quasi-hereditary algebra.

Some corollaries are given below.

For $x, y \geq 0$, consider the graded $(x+y)$-Kronecker quiver with $x+y$ arrows from 1 to 2 , $x$ of which are in degree 0 and $y$ of which are in degree 1 . Let $\Lambda(x, y)$ denote the graded path algebra of this quiver. If $x=y=0$, then $\Lambda(0,0)=B(0,0)$ is the product of two copies of the field $k$. It is known that $H H^{0}(B(0,0))=k \times k$ and $H H^{p}(B(0,0))$ vanishes for $p \geq 1$. In the proof of Theorem 3.6 we have shown that the quasi-hereditary algebra $B(x, y)$ with $x+y \geq 1$ is derived equivalent to $\Lambda(y, x)$. Thus we have by Example 3.5

Corollary 3.8. The dimensions of the Hochschild cohomologies of $B(x, y)$ is given by

$$
\begin{aligned}
& \operatorname{dim}_{k} H H^{p}(B(x, y))=\left\{\begin{array}{ll}
x y+1 & p=0 \\
x^{2}+y^{2}-1 & p=1 \\
x y & p=2 \\
0 & \text { otherwise }
\end{array} \quad \text { if } x+y \geq 1,\right. \\
& \operatorname{dim}_{k} H H^{p}(B(0,0))= \begin{cases}2 & p=0 \\
0 & \text { otherwise. }\end{cases}
\end{aligned}
$$

There is the following sufficient condition for derived simplicity for finite-dimensional algebras with two simple modules and of finite global dimension.

Corollary 3.9. Let $k$ be algebraically closed and $A$ a finite-dimensional $k$-algebra with two simple modules. Assume that $A$ has finite global dimension. If $H H^{p}(A) \neq 0$ for some $p>2$, then $A$ is derived simple.

Proof. Suppose that $A$ is not derived simple. Then by Theorem 3.7, $A$ is derived equivalent to some quasi-hereditary algebra with two simples, i.e. to some $B(x, y)$. By Corollary 3.8 , the space $H H^{p}(A)$ vanishes for all $p>2$.

Corollary 3.10. There are natural one-to-one correspondences among the following sets:

(i) Morita equivalence classes of quasi-hereditary algebras with two simple modules whose endomorphism algebras are isomorphic to $k$, modulo Ringel duality;

(ii) $B(x, y)$ for $y \geq x \geq 0$;

(iii) graded $n$-Kronecker algebras $\Lambda(x, y)$ for $x \geq y \geq 0$;

(iv) derived equivalence classes of finite-dimensional algebras with two simple modules such that the derived category admits a stratification with all simple factors being $k$. 
Proof. [(ii) $\leftrightarrow($ iii)] The map $B(x, y) \mapsto \Lambda(y, x)$ is one-to-one and onto.

$[($ ii) $\leftrightarrow$ (iv)] It follows from Theorem 3.6 that in each derived equivalence class in (iv) there is an algebra of the form $B(x, y)$ for some $x, y \geq 0$. Since $B(x, y)$ and $B(y, x)$ are derived equivalent, we can choose $x$ and $y$ such that $y \geq x$. It remains to show that $B\left(x^{\prime}, y^{\prime}\right)$ is derived equivalent to $B(x, y)$ if and only if $\left(x^{\prime}, y^{\prime}\right)=(x, y)$ or $\left(x^{\prime}, y^{\prime}\right)=(y, x)$. Suppose that $B(x, y)$ and $B\left(x^{\prime}, y^{\prime}\right)$ are derived equivalent. Then they have isomorphic Hochschild cohomologies. Thus by Corollary 3.8 we have

$$
\left\{\begin{array}{l}
x y+1=x^{\prime} y^{\prime}+1 \\
x^{2}+y^{2}-1=x^{2}+y^{\prime 2}-1 \quad \text { or } \quad x=y=0=x^{\prime}=y^{\prime} . \\
x y=x^{\prime} y^{\prime}
\end{array}\right.
$$

Solving this equation we obtain $\left(x^{\prime}, y^{\prime}\right)=(x, y)$ or $\left(x^{\prime}, y^{\prime}\right)=(y, x)$.

$[(\mathrm{i}) \leftrightarrow(\mathrm{ii})]$ First, in each Morita equivalence class in (i) there is an algebra of the form $B(x, y)$ for some $x, y \geq 0$. Secondly, as remarked in Section 2.4, the algebras $B(x, y)$ and $B(y, x)$ are Ringel dual to each other. Finally, if $y \geq x \geq 0, y^{\prime} \geq x^{\prime} \geq 0$ and $(x, y) \neq\left(x^{\prime}, y^{\prime}\right)$, then $B(x, y)$ and $B\left(x^{\prime}, y^{\prime}\right)$ are not derived equivalent, and hence they are not Morita equivalent.

\section{An example}

Let $k$ be algebraically closed. In this section, we construct a family $A_{n}(\underline{x}, \underline{y})(n \geq 0, \underline{x}, \underline{y}$ are sequences of positive integers) of algebras with two simples. We will show that if $n \leq 2$ then the algebra $A_{n}(\underline{x}, \underline{y})$ is isomorphic to a quasi-hereditary algebra; otherwise, it is derived simple.

When $\underline{x}=\underline{y}=(1,1, \ldots)$, the algebra $A_{n}(\underline{x}, \underline{y})$ is the same as the Fibonacci algebra $A_{n}$ constructed by E. Green [10] and further studied by Happel [13] and Membrillo-Hernández [18]. In 13, 18, it is shown that $A_{n}(n \geq 3)$ is not derived equivalent to quasi-hereditary algebras. The method used in [18] works for our general $A_{n}(\underline{x}, \underline{y})$ with $n$ even but not for $n$ odd. In this section we provide a different method, which uses the top Hochschild cohomology.

4.1. The construction. Let $\underline{x}=\left(x_{1}, x_{2}, \ldots\right), \underline{y}=\left(y_{1}, y_{2}, \ldots\right)$ be two sequences of positive integers. We define a family of algebras $A_{n}(\underline{x}, \underline{y})$ recursively as follows.

$A_{0}(\underline{x}, \underline{y})$ is the path algebra of the quiver with two vertices 1 and 2 and without arrows. Namely, $A_{0}(\underline{x}, \underline{y})=k \oplus k$.

$A_{1}(\underline{x}, \underline{y})$ is the path algebra of the $x_{1}$-Kronecker quiver: it has two vertices 1 and 2 , and has $x_{1}$ arrows, all of which go from 1 to 2 .

For $m \geq 1$, the algebra $A_{2 m}(\underline{x}, \underline{y})$ is obtained from $A_{2 m-1}(\underline{x}, \underline{y})$ by adding $y_{m}$ new arrows $\beta_{m, j}\left(1 \leq j \leq y_{m}\right)$ from 2 to 1 and adding as new relations the paths $\beta_{m, j} \alpha_{i^{\prime}, j^{\prime}}\left(1 \leq j \leq y_{m}\right.$, $1 \leq i^{\prime} \leq m$ and $\left.1 \leq j^{\prime} \leq x_{i^{\prime}}\right)$; the algebra $A_{2 m+1}(\underline{x}, \underline{y})$ is obtained from $A_{2 m}(\underline{x}, \underline{y})$ by adding $x_{m+1}$ new arrows $\alpha_{m+1, j}\left(1 \leq j \leq x_{m+1}\right)$ from 1 and 2 and adding as new relations the paths $\alpha_{m+1, j} \beta_{i^{\prime}, j^{\prime}}\left(1 \leq j \leq x_{m+1}, 1 \leq i^{\prime} \leq m\right.$ and $\left.1 \leq j^{\prime} \leq y_{i^{\prime}}\right)$.

In other words, the quiver of $A_{n}(\underline{x}, \underline{y})$ has two vertices 1 and 2 and has $x_{1}+\ldots+x_{\left\lfloor\frac{n+1}{2}\right\rfloor}$ arrows from 1 to 2 labelled by $\alpha_{i, j}\left(1 \leq i \leq\left\lfloor\frac{n+1}{2}\right\rfloor\right.$ and $\left.1 \leq j \leq x_{i}\right)$ and $y_{1}+\ldots+y_{\left\lfloor\frac{n}{2}\right\rfloor}$ arrows labelled by $\beta_{i, j}\left(1 \leq i \leq\left\lfloor\frac{n}{2}\right\rfloor\right.$ and $\left.1 \leq j \leq y_{i}\right)$. Here for a real number $r$ we denote by $\lfloor r\rfloor$ the greatest 
integer smaller than or equal to $r$. The relations of $A_{n}(\underline{x}, \underline{y})$ are $\alpha_{i, j} \beta_{i^{\prime}, j^{\prime}}\left(1 \leq i^{\prime}<i \leq\left\lfloor\frac{n+1}{2}\right\rfloor\right.$, $1 \leq j \leq x_{i}$ and $\left.1 \leq j^{\prime} \leq y_{i^{\prime}}\right)$ and $\beta_{i^{\prime}, j^{\prime}} \alpha_{i, j}\left(1 \leq i \leq i^{\prime} \leq\left\lfloor\frac{n}{2}\right\rfloor, 1 \leq j^{\prime} \leq y_{i^{\prime}}\right.$ and $\left.1 \leq j \leq x_{i}\right)$.

Notice that $A_{1}(\underline{x}, \underline{y})=B\left(x_{1}, 0\right) \cong B\left(0, x_{1}\right)$ and $A_{2}(\underline{x}, \underline{y})=B\left(x_{1}, y_{1}\right)$. Thus (up to Morita equivalence) the set $\left\{A_{n}(\underline{x}, \underline{y}) \mid n \leq 2, \underline{x}, \underline{y}\right.$ arbitrary $\}$ consists of all quasi-hereditary algebras with two simple modules.

4.2. The Cartan matrix. Let $e_{1}, e_{2}$ be the two primitive idempotents of the algebra $A_{n}(\underline{x}, \underline{y})$. Recall that the Cartan matrix $C_{n}(\underline{x}, \underline{y})=\left(c_{i j}\right)$ of $A_{n}(\underline{x}, \underline{y})$ is the $2 \times 2$ matrix with entries $c_{i j}=\operatorname{dim}_{k}\left(e_{i} A e_{j}\right)$, which count precisely the paths starting from $j$ and ending at $i$. One can check directly that

$$
\begin{aligned}
C_{0}(\underline{x}, \underline{y}) & =\left(\begin{array}{ll}
1 & 0 \\
0 & 1
\end{array}\right), \\
C_{1}(\underline{x}, \underline{y}) & =\left(\begin{array}{ll}
1 & 0 \\
x_{1} & 1
\end{array}\right), \\
C_{2}(\underline{x}, \underline{y}) & =\left(\begin{array}{cc}
1 & y_{1} \\
x_{1} & 1+x_{1} y_{1}
\end{array}\right), \\
C_{3}(\underline{x}, \underline{y}) & =\left(\begin{array}{cc}
1+y_{1} x_{2} & y_{1} \\
x_{1}+x_{2}+x_{1} y_{1} x_{2} & 1+x_{1} y_{1}
\end{array}\right), \\
C_{4}(\underline{x}, \underline{y}) & =\left(\begin{array}{cc}
1+y_{1} x_{2} & y_{1}+y_{2}+y_{1} x_{2} y_{2} \\
x_{1}+x_{2}+x_{1} y_{1} x_{2} & 1+x_{1} y_{1}+x_{1} y_{2}+x_{2} y_{2}+x_{1} y_{1} x_{2} y_{2}
\end{array}\right) .
\end{aligned}
$$

We will use the following 'generalized Fibonacci numbers'

$$
F(\underline{x}, \underline{y})=\left(F_{0}(\underline{x}, \underline{y}), F_{1}(\underline{x}, \underline{y}), F_{2}(\underline{x}, \underline{y}), \ldots\right)
$$

to describe $C_{n}(\underline{x}, \underline{y})$ : Here $F_{0}(\underline{x}, \underline{y})=0, F_{1}(\underline{x}, \underline{y})=1$ and inductively

$$
\begin{aligned}
F_{2 m}(\underline{x}, \underline{y}) & =F_{2 m-2}(\underline{x}, \underline{y})+F_{2 m-1}(\underline{x}, \underline{y}) x_{m}, \\
F_{2 m+1}(\underline{x}, \underline{y}) & =F_{2 m-1}(\underline{x}, \underline{y})+F_{2 m}(\underline{x}, \underline{y}) y_{m} .
\end{aligned}
$$

Indeed, $F_{2}(\underline{x}, \underline{y})=x_{1}, F_{3}(\underline{x}, \underline{y})=1+x_{1} y_{1}, F_{4}(\underline{x}, \underline{y})=x_{1}+x_{2}+x_{1} y_{1} x_{2}, F_{5}(\underline{x}, \underline{y})=1+x_{1} y_{1}+$ $x_{1} y_{2}+x_{2} y_{2}+x_{1} y_{1} x_{2} y_{2}$. When $\underline{x}=\underline{y}=(1,1, \ldots)$, the generalised Fibonacci numbers are exactly the usual Fibonacci numbers.

The following lemma can be proved inductively. For a sequence $\underline{x}$, let $S \underline{x}=\left(x_{2}, x_{3}, \ldots\right)$ denote its shift.

Lemma 4.1. The following formulas hold
(1) $C_{2 m}(\underline{x}, \underline{y})=\left(\begin{array}{cc}1 & y_{m} \\ 0 & 1\end{array}\right) \times C_{2 m-1}(\underline{x}, \underline{y})=\left(\begin{array}{cc}F_{2 m-1}(\underline{y}, S \underline{x}) & F_{2 m}(\underline{y}, S \underline{x}) \\ F_{2 m}(\underline{x}, \underline{y}) & F_{2 m+1}(\underline{x}, \underline{y})\end{array}\right)$.
(2) $C_{2 m+1}(\underline{x}, \underline{y})=\left(\begin{array}{cc}1 & 0 \\ x_{m+1} & 1\end{array}\right) \times C_{2 m}(\underline{x}, \underline{y})=\left(\begin{array}{cc}F_{2 m+1}(\underline{y}, S \underline{x}) & F_{2 m}(\underline{y}, S \underline{x}) \\ F_{2 m+2}(\underline{x}, \underline{y}) & F_{2 m+1}(\underline{x}, \underline{y})\end{array}\right)$. 
4.3. The quadratic dual. Fix $n \geq 0$ and two sequences of positive integers $\underline{x}, \underline{y}$. Let $A=$ $A_{n}(\underline{x}, \underline{y})$.

The quadratic dual $A^{!}$of $A$ is given by the same quiver as $A$ with relations $\beta_{i^{\prime}, j^{\prime}} \alpha_{i, j}(1 \leq$ $i^{\prime}<i \leq\left\lfloor\frac{n+1}{2}\right\rfloor, 1 \leq j^{\prime} \leq y_{i^{\prime}}$ and $\left.1 \leq j \leq x_{i}\right)$ and $\alpha_{i, j} \beta_{i^{\prime}, j^{\prime}}\left(1 \leq i \leq i^{\prime} \leq\left\lfloor\frac{n}{2}\right\rfloor, 1 \leq j \leq x_{i}\right.$ and $\left.1 \leq j^{\prime} \leq y_{i^{\prime}}\right)$. We remark that this is opposite to the usual definition but it is more convenient for our purpose.

The algebra $A^{!}$is naturally graded by path length. One checks that the degree $p$ component $\left(A^{!}\right)^{p}$ vanishes for $p>n$; the degree $n$ component $\left(A^{!}\right)^{n}$ has a basis

$$
\left\{\beta_{m, j_{m}^{\prime}} \alpha_{m, j_{m}} \cdots \beta_{2, j_{2}^{\prime}} \alpha_{2, j_{2}} \beta_{1, j_{1}^{\prime}} \alpha_{1, j_{1}} \mid 1 \leq j_{i}^{\prime} \leq y_{i} \text { and } 1 \leq j_{i} \leq x_{i}\right\}
$$

for $n=2 m$ and

$$
\left\{\alpha_{m+1, j_{m+1}} \beta_{m, j_{m}^{\prime}} \alpha_{m, j_{m}} \cdots \beta_{2, j_{2}^{\prime}} \alpha_{2, j_{2}} \beta_{1, j_{1}^{\prime}} \alpha_{1, j_{1}} \mid 1 \leq j_{i}^{\prime} \leq y_{i} \text { and } 1 \leq j_{i} \leq x_{i}\right\}
$$

for $n=2 m+1$; the degree $n-1$ component $\left(A^{!}\right)^{n-1}$ has a basis

$$
\begin{array}{r}
\left\{\alpha_{m, j_{m}} \cdots \beta_{2, j_{2}^{\prime}} \alpha_{2, j_{2}} \beta_{1, j_{1}^{\prime}} \alpha_{1, j_{1}} \mid 1 \leq j_{i}^{\prime} \leq y_{i} \text { and } 1 \leq j_{i} \leq x_{i}\right\} \\
\cup\left\{\beta_{m, j_{m}^{\prime}} \alpha_{m, j_{m}} \cdots \beta_{2, j_{2}^{\prime}} \alpha_{2, j_{2}} \beta_{1, j_{1}^{\prime}} \mid 1 \leq j_{i}^{\prime} \leq y_{i} \text { and } 1 \leq j_{i} \leq x_{i}\right\}
\end{array}
$$

for $n=2 m$ and

$$
\begin{array}{r}
\left\{\beta_{m, j_{m}^{\prime}} \alpha_{m, j_{m}} \cdots \beta_{2, j_{2}^{\prime}} \alpha_{2, j_{2}} \beta_{1, j_{1}^{\prime}} \alpha_{1, j_{1}} \mid 1 \leq j_{i}^{\prime} \leq y_{i} \text { and } 1 \leq j_{i} \leq x_{i}\right\} \\
\cup\left\{\alpha_{m+1, j_{m+1}} \beta_{m, j_{m}^{\prime}} \alpha_{m, j_{m}} \cdots \beta_{2, j_{2}^{\prime}} \alpha_{2, j_{2}} \beta_{1, j_{1}^{\prime}} \mid 1 \leq j_{i}^{\prime} \leq y_{i} \text { and } 1 \leq j_{i} \leq x_{i}\right\}
\end{array}
$$

for $n=2 m+1$.

By definition, the algebra $A$ is a quadratic monomial algebra, and hence Koszul by [11, Proposition 2.2]. Therefore, by [3, Theorem 2.10.1] (Reminder: we are considering right modules), there is a graded algebra isomorphism between the opposite algebra of $A^{!}$and the Yoneda algebra $\bigoplus_{p \geq 0} \operatorname{Ext}_{A}^{p}\left(S_{1} \oplus S_{2}, S_{1} \oplus S_{2}\right)$, where $S_{1}, S_{2}$ are the two simple modules of $A$. It follows that the global dimension of $A$ equals $n$.

4.4. The top Hochschild cohomology. Fix $n \geq 0$ and two sequences of positive integers $\underline{x}, \underline{y}$. Let $A=A_{n}(\underline{x}, \underline{y})$. Since the global dimension of $A$ is $n$, it follows that $H H^{p}(A)$ vanishes for $p>n$ (see Section 2.2). In this subsection, we show that $H H^{n}(A) \neq 0$. For the case $\underline{x}=\underline{y}=(1,1,1, \ldots)$, the Hochschild cohomologies of $A$ together with the algebra structure of $\bigoplus_{p \in \mathbb{Z}} H H^{p}(A)$ were determined by Fan and $\mathrm{Xu}$ in $[8,9]$.

Recall that $A$ is Koszul. Then the Koszul bimodule complex provides a projective resolution of $A$ as a bimodule, by [21, Section 3]. Since we compute $H H^{n}$ only, it suffices to write down the components in degrees $-n$ and $-n+1$ :

$$
A \otimes_{k Q_{0}}\left(A^{!}\right)^{n} \otimes_{k Q_{0}} A \stackrel{f}{\longrightarrow} A \otimes_{k Q_{0}}\left(A^{!}\right)^{n-1} \otimes_{k Q_{0}} A
$$


Then $H H^{n}(A)$ is the cokernel of the homomorphism $f^{*}=\operatorname{Hom}_{A^{e}}(f, A)$. There are isomorphisms

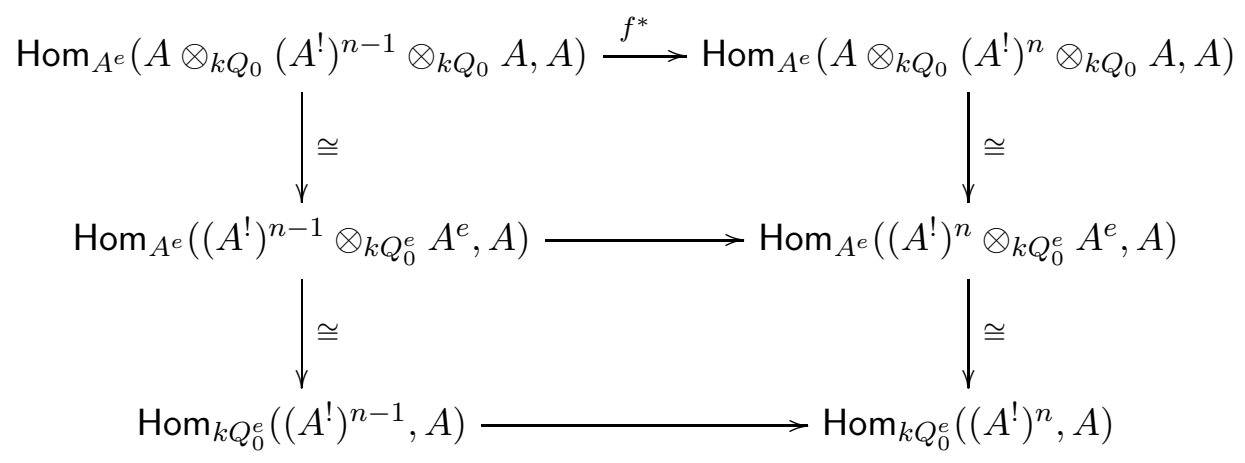

where the map on the last row is given by

$$
f^{*}(\varphi): \rho_{1} \cdots \rho_{n} \mapsto \varphi\left(\rho_{1} \cdots \rho_{n-1}\right) \rho_{n}-\rho_{1} \varphi\left(\rho_{2} \cdots \rho_{n}\right)
$$

for $\varphi:\left(A^{!}\right)^{n-1} \rightarrow A$ and basis elements $\rho_{1} \cdots \rho_{n} \in\left(A^{!}\right)^{n}$.

Proposition 4.2. Assume $n \geq 2$. Then the dimension of the top Hochschild cohomology of $A$ is given by

$$
\operatorname{dim}_{k} H H^{n}(A)= \begin{cases}F_{n-1}(\underline{y}, S \underline{x}) \prod_{i=1}^{\frac{n}{2}} x_{i} y_{i} & \text { if } n \text { is even } \\ F_{n+1}(\underline{x}, \underline{y}) x_{\frac{n+1}{2}} \prod_{i=1}^{\frac{n-1}{2}} x_{i} y_{i}-2 & \text { if } n \text { is odd } .\end{cases}
$$

Proof. For $n=2 m$ : Recall from Section 4.3 that $\left(A^{!}\right)^{n}$ has a basis $\left\{\beta_{m} \alpha_{m} \cdots \beta_{2} \alpha_{2} \beta_{1} \alpha_{1}\right\}$ and $\left(A^{!}\right)^{n-1}$ has a basis $\left\{\alpha_{m} \cdots \beta_{2} \alpha_{2} \beta_{1} \alpha_{1}\right\} \cup\left\{\beta_{m} \alpha_{m} \cdots \beta_{2} \alpha_{2} \beta_{1}\right\}$, where $\beta_{i}$ ranges over $\left\{\beta_{i, 1}, \ldots, \beta_{i, y_{i}}\right\}$ and $\alpha_{i}$ ranges over $\left\{\alpha_{i, 1}, \ldots, \alpha_{i, x_{i}}\right\}$ for all $1 \leq i \leq m$. All these basis elements of $\left(A^{!}\right)^{n}$ are cycles at 1 . Therefore,

$$
\operatorname{dim}_{k} \operatorname{Hom}_{k Q_{0}^{e}}\left(\left(A^{!}\right)^{n}, A\right)=\operatorname{dim}_{k}\left(e_{1} A e_{1}\right)^{\oplus \prod_{i=1}^{m} x_{i} y_{i}}=F_{n-1}(\underline{y}, S \underline{x}) \prod_{i=1}^{m} x_{i} y_{i} .
$$

Here $\prod_{i=1}^{m} x_{i} y_{i}$ is the dimension of $\left(A^{!}\right)^{n}$, and $\operatorname{dim}_{k}\left(e_{1} A e_{1}\right)$ is the $(1,1)$-entry in the Cartan matrix, namely, $F_{n-1}(\underline{y}, S \underline{x})$ by Lemma 4.1. The path $\beta_{m} \alpha_{m} \cdots \beta_{2} \alpha_{1} \beta_{1}$ in $\left(A^{!}\right)^{n-1}$ starts from 2 and ends at 1 , so a morphism $\varphi \in \operatorname{Hom}_{k Q_{0}^{e}}\left(\left(A^{!}\right)^{n-1}, A\right)$ maps it to a linear combination of paths in $A$ starting from 2 and ending at 1 . It follows that $\varphi\left(\beta_{m} \alpha_{m} \cdots \beta_{2} \alpha_{1} \beta_{1}\right) \alpha_{1}=0$ for all $\varphi \in \operatorname{Hom}_{k Q_{0}^{e}}\left(\left(A^{!}\right)^{n-1}, A\right)$. Similarly, $\beta_{m} \varphi\left(\alpha_{m} \cdots \beta_{2} \alpha_{2} \beta_{1} \alpha_{1}\right)=0$ for all $\varphi \in \operatorname{Hom}_{k Q_{0}^{e}}\left(\left(A^{!}\right)^{n-1}, A\right)$. We have shown that $f^{*}: \operatorname{Hom}_{k Q_{0}^{e}}\left(\left(A^{!}\right)^{n-1}, A\right) \rightarrow \operatorname{Hom}_{k Q_{0}^{e}}\left(\left(A^{!}\right)^{n}, A\right)$ is trivial. Therefore,

$$
\operatorname{dim}_{k} H H^{n}(A)=\operatorname{dim}_{k} \operatorname{Hom}_{k Q_{0}^{e}}\left(\left(A^{!}\right)^{n}, A\right)=F_{n-1}(\underline{y}, S \underline{x}) \prod_{i=1}^{m} x_{i} y_{i},
$$

as desired.

For $n=2 m+1$ : Recall from Section 4.3 that $\left(A^{!}\right)^{n}$ has a basis $\left\{\alpha_{m+1} \beta_{m} \alpha_{m} \cdots \beta_{2} \alpha_{2} \beta_{1} \alpha_{1}\right\}$ and $\left(A^{!}\right)^{n-1}$ has a basis $\left\{\beta_{m} \alpha_{m} \cdots \beta_{2} \alpha_{2} \beta_{1} \alpha_{1}\right\} \cup\left\{\alpha_{m+1} \beta_{m} \alpha_{m} \cdots \beta_{2} \alpha_{2} \beta_{1}\right\}$, where $\beta_{i}$ ranges over $\left\{\beta_{i, 1}, \ldots, \beta_{i, y_{i}}\right\}$ for all $1 \leq i \leq m$ and $\alpha_{i}$ ranges over $\left\{\alpha_{i, 1}, \ldots, \alpha_{i, x_{i}}\right\}$ for all $1 \leq i \leq m+1$. Therefore,

$$
\operatorname{dim}_{k} \operatorname{Hom}_{k Q_{0}^{e}}\left(\left(A^{!}\right)^{n}, A\right)=\operatorname{dim}_{k}\left(e_{2} A e_{1}\right)^{\oplus x_{m+1} \prod_{i=1}^{m} x_{i} y_{i}}=F_{n+1}(\underline{x}, \underline{y}) x_{m+1} \prod_{i=1}^{m} x_{i} y_{i} .
$$


The path $\alpha_{m+1} \beta_{m} \alpha_{m} \cdots \beta_{2} \alpha_{2} \beta_{1}$ of $\left(A^{!}\right)^{n-1}$ is a cycle at 2 . A morphism $\varphi \in \operatorname{Hom}_{k Q_{0}^{e}}\left(A^{!}\right)^{n-1} \rightarrow A$ maps it to a linear combination of cycles of $A$ at 2 . All such cycles become trivial when composed with $\alpha_{1}$ on the right, except the trivial circle $e_{2}$. So

$$
\varphi\left(\alpha_{m+1} \beta_{m} \alpha_{m} \cdots \beta_{2} \alpha_{1} \beta_{1}\right) \alpha_{1} \neq 0 \Longleftrightarrow \varphi\left(\alpha_{m+1} \beta_{m} \alpha_{m} \cdots \beta_{2} \alpha_{1} \beta_{1}\right)=e_{2} .
$$

Similarly,

$$
\alpha_{m+1} \varphi\left(\beta_{m} \alpha_{m} \cdots \beta_{2} \alpha_{2} \beta_{1} \alpha_{1}\right) \neq 0 \Longleftrightarrow \varphi\left(\beta_{m} \alpha_{m} \cdots \beta_{2} \alpha_{2} \beta_{1} \alpha_{1}\right)=e_{1} .
$$

We have shown that the image of $f^{*}: \operatorname{Hom}_{k Q_{0}^{e}}\left(\left(A^{!}\right)^{n-1}, A\right) \rightarrow \operatorname{Hom}_{k Q_{0}^{e}}\left(\left(A^{!}\right)^{n}, A\right)$ has dimension 2. Therefore

$$
\operatorname{dim}_{k} H H^{n}(A)=\operatorname{dim}_{k} \operatorname{Hom}_{k Q_{0}^{e}}\left(\left(A^{!}\right)^{n}, A\right)-2=F_{n+1}(\underline{x}, \underline{y}) x_{m+1} \prod_{i=1}^{m} x_{i} y_{i}-2,
$$

as desired.

Recall that $A_{1}(\underline{x}, \underline{y})=B\left(x_{1}, 0\right)$ and $A_{0}(\underline{x}, \underline{y})=B(0,0)$. Thus by Corollary [3.8, we have $\operatorname{dim}_{k} H H^{1}\left(A_{1}(\underline{x}, \underline{y})\right)=x_{1}^{2}-1, H H^{0}\left(A_{1}(\underline{x}, \underline{y})\right)=k$ and $H H^{0}\left(A_{0}(\underline{x}, \underline{y})\right)=k \oplus k$.

4.5. Derived simplicity. By combining the results in the previous sections we obtain the derived simplicity of the algebras $A_{n}(\underline{x}, \underline{y})$ for all $n \geq 3$.

Corollary 4.3. Let $\underline{x}, \underline{y}, \underline{x}^{\prime}, \underline{y}^{\prime}$ be four sequences of positive integers and $n, n^{\prime}$ two non-negative integers. If $n \neq n^{\prime}$, then the algebras $A_{n}(\underline{x}, \underline{y})$ and $A_{n^{\prime}}\left(\underline{x}^{\prime}, \underline{y}^{\prime}\right)$ are not derived equivalent. Furthermore, when $n \geq 3$, the algebras $A_{n}(\underline{x}, \underline{y})$ are all derived simple.

Proof. To prove the first statement, let us suppose $n>n^{\prime}$. If $n \geq 2$, then $H H^{n}\left(A_{n}(\underline{x}, \underline{y})\right) \neq 0$ by Proposition 4.2, while $H H^{n}\left(A_{n^{\prime}}\left(\underline{x}^{\prime}, \underline{y}^{\prime}\right)\right)=0$ because $n>n^{\prime}=\operatorname{gl} \cdot \operatorname{dim} A_{n^{\prime}}\left(\underline{x}^{\prime}, \underline{y}^{\prime}\right)$. So $A_{n}(\underline{x}, \underline{y})$ and $A_{n^{\prime}}\left(\underline{x}^{\prime}, \underline{y}^{\prime}\right)$ are not derived equivalent. If $n=1$ and $n^{\prime}=0$, then $A_{n^{\prime}}\left(\underline{x}^{\prime}, \underline{y}^{\prime}\right)$ is semisimple, while $A_{n}(\underline{x}, \underline{y})$ is not semisimple, so they are not derived equivalent.

In Section 4.3 we have already shown that $A_{n}(\underline{x}, \underline{y})$ has finite global dimension, whence it is derived simple or derived equivalent to a quasi-hereditary algebra by Theorem 3.7. Thus the second statement is an immediate consequence of the first one, since up to Morita equivalence all quasi-hereditary algebras with two simple modules belong to the set $\left\{A_{n}(\underline{x}, \underline{y}) \mid n \leq\right.$ $2, \underline{x}, \underline{y}$ arbitrary\}.

4.6. Further properties. In this subsection, we list some further properties of $A_{n}(\underline{x}, \underline{y})$ and $A_{n}(\underline{x}, \underline{y})^{!}$and leave the proof to the interested reader. We denote by $S_{1}, S_{2}$ and $P_{1}, P_{2}$ the simple and indecomposable projective modules corresponding to the vertices 1 and 2 , respectively.

Lemma 4.4. Fix two sequences of positive integers $\underline{x}, \underline{y}$.

(1) The socle of $A_{2 m}(\underline{x}, \underline{y})$ is a direct sum of copies of $S_{2}$; the socle of $A_{2 m+1}(\underline{x}, \underline{y})$ is a direct sum of copies of $S_{1}$.

(2) The indecomposable projective modules of $A_{2 m}(\underline{x}, \underline{y})$ are obtained from those of $A_{2 m-1}(\underline{x}, \underline{y})$, by adding $y_{m}$ copies of $S_{2}$ to each subfactor $S_{1}$.

(3) The indecomposable projective modules of $A_{2 m+1}(\underline{x}, \underline{y})$ are obtained from those of $A_{2 m}(\underline{x}, \underline{y})$, by adding $x_{m+1}$ copies of $S_{1}$ to each subfactor $S_{2}$. 
(4) The socle of $A_{n}(\underline{x}, \underline{y})^{!}$is a direct sum of copies of the simple module $S_{1}$.

(5) The module $P_{2}$ over $A_{2 m}(\underline{x}, \underline{y})^{!}$is the same as over $A_{2 m-1}(\underline{x}, \underline{y})^{!}$; the module $P_{1}$ over $A_{2 m}(\underline{x}, \underline{y})^{!}$is obtained from the $P_{1}$ over $A_{2 m-1}(\underline{x}, \underline{y})^{!}$by adding $y_{m}$ copies of $P_{2}$ to the top of $P_{1}$.

(6) The module $P_{1}$ over $A_{2 m+1}^{!}(\underline{x}, \underline{y})$ is the same as $P_{1}$ over $A_{2 m}^{!}(\underline{x}, \underline{y})$; the module $P_{2}$ over $A_{2 m+1}^{!}(\underline{x}, \underline{y})$ is obtained from the $P_{2}$ over $A_{2 m}^{!}(\underline{x}, \underline{y})$ by adding $x_{m+1}$ copies of $P_{1}$ to the top of $P_{2}$.

\section{REFERENCES}

[1] Takuma Aihara and Osamu Iyama, Silting mutation in triangulated categories, J. Lond. Math. Soc. (2) 85 (2012), no. 3, 633-668.

[2] Lidia Angeleri Hügel, Steffen Koenig, Qunhua Liu, and Dong Yang, Derived simple algebras and restrictions of recollements of derived module categories, preprint (2013), arXiv:1310.3479

[3] Alexander Beilinson, Victor Ginzburg, and Wolfgang Soergel, Koszul duality patterns in representation theory, J. Amer. Math. Soc. 9 (1996), no. 2, 473-527.

[4] Alexander A. Beilinson, Joseph Bernstein, and Pierre Deligne, Faisceaux pervers, Astérisque, vol. 100, Soc. Math. France, 1982 (French).

[5] Edward Cline, Brian Parshall, and Leonard L. Scott, Algebraic stratification in representation categories, J. Algebra 117 (1988), no. 2, 504-521.

[6] _ Finite-dimensional algebras and highest weight categories, J. reine ang. Math. 391 (1988), 85-99.

[7] Vlastimil Dlab and Claus Michael Ringel, Quasi-hereditary algebras, Illinois J. Math. 33 (1989), 280-291.

[8] Jinmei Fan and Yunge Xu, On Hochschild cohomology rings of Fibonacci algebras, Front. Math. China 1 (2006), no. 4, 526-537.

[9] _ Hochschild cohomology groups of Fibonacci algebras, Chin. Ann. Math. Ser. A 28 (2007), no. 3 (Chinese).

[10] Edward L. Green, Remarks on projective resolutions, Representation theory, II (Proc. Second Internat. Conf., Carleton Univ., Ottawa, Ont., 1979), Lecture Notes in Math., vol. 832, Springer, Berlin, 1980, pp. 259-279.

[11] Edward L. Green and Dan Zacharia, The cohomology ring of a monomial algebra, Manuscripta Math. 85 (1994), 11-23.

[12] Dieter Happel, Hochschild cohomology of finite-dimensional algebras, Séminaire d'Algèbre Paul Dubreil et Marie-Paul Malliavin, 39ème Année (Paris, 1987/1988), Lecture Notes in Math., vol. 1404, Springer, Berlin, 1989, pp. 108-126.

[13] _ A family of algebras with two simple modules and Fibonacci numbers, Arch. Math. (Basel) 57 (1991), no. 2, 133-139.

[14] Bernhard Keller, Derived invariance of higher structures on the Hochschild complex, preprint, 2003.

[15] _ Deriving DG categories, Ann. Sci. École Norm. Sup. (4) 27 (1994), no. 1, 63-102.

[16] _ On differential graded categories, International Congress of Mathematicians. Vol. II, Eur. Math. Soc., Zürich, 2006, pp. 151-190.

[17] Bernhard Keller and Dong Yang, Derived equivalences from mutations of quivers with potential, Adv. Math. 226 (2011), no. 3, 2118-2168.

[18] Fausto H. Membrillo-Hernández, Quasi-hereditary algebras with two simple modules and Fibonacci numbers, Commun. Algebra 22 (1994), no. 11, 4499-4509.

[19] Claus Michael Ringel, The category of modules with good filtration over a quasi-hereditary algebra has almost split sequences, Math. Z. 208 (1991), 209-223.

[20] Leonard L. Scott, Simulating algebraic geometry with algebra. I. The algebraic theory of derived categories, The Arcata Conference on Representations of Finite Groups (Arcata, Calif., 1986), Proc. Sympos. Pure Math., vol. 47, Amer. Math. Soc., Providence, RI, 1987, pp. 271-281. 
[21] Michel Van den Bergh, Non-commutative homology of some three dimensional quantum spaces, J. K-theory 8 (1994), 213-230.

Qunhua Liu, Institute of Mathematics, School of Mathematical Sciences, Nanjing Normal UniVersity, 1 Wenyuan Road, Yadong New District, Nanjing 210023, P.R.China

E-mail address: 05402@njnu.edu.cn

Dong Yang, Department of Mathematics, Nanjing University, 22 Hankou Road, Nanjing 210093, P. R. CHINA

E-mail address: dongyang2002@gmail.com 